Daniel Cher, M.D.

Vice President of Clinical and Regulatory Affairs

ev3, Inc.

173 Jefferson Drive

Menlo Park CA 94025-1114

\title{
APR 06011
}

Re: P100018

Pipeline ${ }^{\mathrm{TM}}$ Embolization Device

Filed: May 18, 2010

Amended: July 30, September 17, October 22, November 8 and 15, 2010, and January 12, 2011

Procode: OUT (Intracranial Aneurysm Flow Diverter)

Dear Dr. Cher:

The Center for Devices and Radiological Health (CDRH) of the Food and Drug Administration (FDA) has completed its review of your premarket approval application (PMA) for the Pipeline ${ }^{\mathrm{TM}}$ Embolization Device. This device is indicated for the endovascular treatment of adults (age 22 and above) with large or giant wide-necked intracranial aneurysms in the internal carotid artery from the petrous to the superior hypophyseal segments. We are pleased to inform you that the PMA is approved. You may begin commercial distribution of the device in accordance with the conditions of approval described below.

The sale and distribution of this device are restricted to prescription use in accordance with 21 CFR 801.109 and under section 515(d)(1)(B)(ii) of the Federal Food, Drug, and Cosmetic Act (the act). The device is further restricted under section $515(\mathrm{~d})(1)(\mathrm{B})(\mathrm{ii})$ of the act insofar as the labeling must specify the specific training or experience practitioners need in order to use the device.

Expiration dating for this device has been established and approved at 3 years This is to advise you that the protocol you used to establish this expiration dating is considered an approved protocol for the purpose of extending the expiration dating as provided by 21 CFR 814.39(a)(7).

Continued approval of this PMA is contingent upon the submission of periodic reports, required under 21 CFR 814.84, at intervals of one year (unless otherwise specified) from the date of approval of the original PMA. Two copies of this report, identified as "Annual Report" and bearing the applicable PMA reference number, should be submitted to the address below. The Annual Report should indicate the beginning and ending date of the period covered by the report and should include the information required by 21 CFR 814.84 . 
In addition to the above, and in order to provide continued reasonable assurance of the safety and effectiveness of the device, the Annual Report must include, separately for each model number (if applicable), the number of devices sold and distributed during the reporting period, including those distributed to distributors. The distribution data will serve as a denominator and provide necessary context for FDA to ascertain the frequency and prevalence of adverse events, as FDA evaluates the continued safety and effectiveness of the device.

In addition to the Annual Report requirements, you must provide the following data in separate post-approval study (PAS) reports. Two copies, identified as "PMA Post-Approval Study Report" and bearing the applicable PMA reference number, should be submitted to the address below. As a condition of approval, you must conduct the following post-approval study:

Continued Follow-up of Premarket Cohorts Study: Per the protocol outline submitted by email dated February 3, 2011 and email correspondence on March 30, 2011, continued follow-up of individuals in the pivotal clinical cohort (PUFS) as well as in the continued access cohort (PUFS-CA) for a total of five years will be conducted to provide additional long-term safety and effectiveness data for patients receiving Pipeline Embolic Device (PED). This prospective, observational, open-label, single-arm cohort study anticipates enrolling 131 subjects from the aforementioned studies and performing clinical exams and angiograms at the 3- and 5-year visits and conducting telephone interviews at the 2- and 4-year visits. A minimum of 70 subjects are required for the primary endpoint analysis, as described below. If less than 70 subjects complete the 5-year follow-up, FDA may require you to enroll new patients or consider other regulatory options to reach the required study sample size. The main study endpoints include: ipsilateral stroke and/or neurovascular death (primary endpoint), complete occlusion of the aneurysm, stenosis of the parent artery, and device-related adverse events. Subgroup analyses will be performed based on the anatomic location of the aneurysm as well as the hypertensive status of the subjects. The study protocol with its stated assumptions has adequate power to test the study hypothesis at 5-years for the overall cohort as well as for subgroups of interest.

Please be advised that the results from these studies should be included in the labeling as these data become available. Any updated labeling must be submitted to FDA in the form of a PMA Supplement.

Be advised that the failure to conduct any such study in compliance with the good clinical laboratory practices in $21 \mathrm{CFR}$ part 58 (if a non-clinical study subject to part 58) or the institutional review board regulations in 21 CFR part 56 and the informed consent regulations in 21 CFR part 50 (if a clinical study involving human subjects) may be grounds for FDA withdrawal of approval of the PMA.

Within 30 days of your receipt of this letter, you must submit a PMA supplement that includes a complete protocol of your post-approval study. Your PMA supplement should be clearly labeled as a "Post-Approval Study Protocol" and submitted in triplicate to the address below. Please reference the PMA number above to facilitate processing. If there are multiple protocols being finalized after PMA approval, please submit each protocol as a separate PMA supplement. For 
more information on post-approval studies, see the FDA guidance document entitled, "Procedures for Handling Post-Approval Studies Imposed by PMA Order" (www.fda.gov/MedicalDevices/DeviceRegulationandGuidance/GuidanceDocuments/ucm070974.h

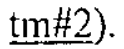

Before making any change affecting the safety or effectiveness of the device, you must submit a PMA supplement or an alternate submission (30-day notice) in accordance with 21 CFR 814.39. All PMA supplements and alternate submissions (30-day notice) must comply with the applicable requirements in 21 CFR 814.39. For more information, please refer to the FDA guidance document entitled, "Modifications to Devices Subject to Premarket Approval (PMA) - The PMA Supplement Decision-Making Process"

(www.fda.gov/MedicalDevices/DeviceRegulationandGuidance/GuidanceDocuments/ucm089274.h $\underline{\mathrm{tm}})$.

You are reminded that many FDA requirements govern the manufacture, distribution, and marketing of devices. For example, in accordance with the Medical Device Reporting (MDR) regulation, 21 CFR 803.50 and 21 CFR 803.52, you are required to report adverse events for this device. Manufacturers of medical devices, including in vitro diagnostic devices, are required to report to FDA no later than 30 calendar days after the day they receive or otherwise becomes aware of information, from any source, that reasonably suggests that one of their marketed devices:

1. May have caused or contributed to a death or serious injury; or

2. Has malfunctioned and such device or similar device marketed by the manufacturer would be likely to cause or contribute to a death or serious injury if the malfunction were to recur.

Additional information on MDR, including how, when, and where to report, is available at www.fda.gov/MedicalDevices/Safety/ReportaProblem/default.htm.

In accordance with the recall requirements specified in 21 CFR 806.10, you are required to submit a written report to FDA of any correction or removal of this device initiated by you to: (1) reduce a risk to health posed by the device; or (2) remedy a violation of the act caused by the device which may present a risk to health, with certain exceptions specified in 21 CFR 806.10(a)(2). Additional information on recalls is available at www.fda.gov/Safety/Recalls/IndustryGuidance/default.htm.

CDRH does not evaluate information related to contract liability warranties. We remind you; however, that device labeling must be truthful and not misleading. CDRH will notify the public of its decision to approve your PMA by making available, among other information, a summary of the safety and effectiveness data upon which the approval is based. The information can be found on the FDA CDRH Internet HomePage located at

www.fda.gov/MedicalDevices/ProductsandMedicalProcedures/DeviceApprovalsandClearances/P MAApprovals/default.htm. Written requests for this information can also be made to the Food and Drug Administration, Dockets Management Branch, (HFA-305), 5630 Fishers Lane, Rm. 1061, Rockville, MD 20852. The written request should include the PMA number or docket number. 
Page 4 - Daniel Cher, M.D.

Within 30 days from the date that this information is placed on the Internet, any interested person may seek review of this decision by submitting a petition for review under section $515(\mathrm{~g})$ of the act and requesting either a hearing or review by an independent advisory committee. FDA may, for good cause, extend this 30-day filing period.

Failure to comply with any post-approval requirement constitutes a ground for withdrawal of approval of a PMA. The introduction or delivery for introduction into interstate commerce of a device that is not in compliance with its conditions of approval is a violation of law.

You are reminded that, as soon as possible and before commercial distribution of your device, you must submit an amendment to this PMA submission with copies of all approved labeling in final printed form. Final printed labeling that is identical to the labeling approved in draft form will not routinely be reviewed by FDA staff when accompanied by a cover letter stating that the final printed labeling is identical to the labeling approved in draft form. If the final printed labeling is not identical, any changes from the final draft labeling should be highlighted and explained in the amendment.

All required documents should be submitted in triplicate, unless otherwise specified, to the address below and should reference the above PMA number to facilitate processing. One of those three copies may be an electronic copy (eCopy), in an electronic format that FDA can process, review and archive (general information:

http://www.fda.gov/MedicalDevices/DeviceRegulationandGuidance/HowtoMarketYourDevice/Pre marketSubmissions/ucm 134508.htm; clinical and statistical data:

http://www.fda.gov/MedicalDevices/DeviceRegulationandGuidance/HowtoMarketYourDevice/Pre marketSubmissions/ucm 136377.htm)

U.S. Food and Drug Administration

Center for Devices and Radiological Health

PMA Document Mail Center - WO66-G609

10903 New Hampshire Avenue

Silver Spring, MD 20993-0002

If you have any questions concerning this approval order, please contact Joseph C. Hutter, Ph.D., at 301-796-6499.

Sincerely yours,

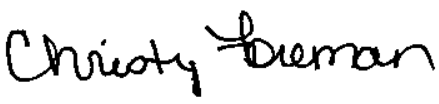

Christy Foreman

Acting Director

Office of Device Evaluation

Center for Devices and Radiological Health

Food and Drug Administration 